difficult asthma clinic were eligible for treatment with omalizumab. There has recently been a change to its prescribing licence with a widening of the eligible range of IgE from $30-700 \mathrm{iu} / \mathrm{ml}$ to $30-1500 \mathrm{iu} / \mathrm{ml}$. Other prescribing criteria are (1) weight between 20 and $150 \mathrm{~kg}$, (2) $\mathrm{FEV}_{1}<80 \%$ predicted, (3) positive skin prick tests or specific IgE to perennial aeroallergens and (4) ongoing symptoms despite a high-dose combination inhaler. In addition NICE guidance now recommends that its use is confined to patients who, within the past year, have had two or more hospital admissions or one admission plus two A\&E attendances for asthma exacerbations. We aimed to determine how the new licensing criteria and the NICE guidelines have affected the proportion of patients attending our difficult asthma clinic eligible for treatment with omalizumab. We assessed 510 patients recording body weight $(\mathrm{kg}), \mathrm{FEV}_{1}(\%$ predicted), symptoms, total $\mathrm{IgE}(\mathrm{iU} / \mathrm{ml})$ and skin prick tests. Our data demonstrate that 240 had an IgE outside the eligible range, 242 patients had an $\mathrm{FEV}_{1}>80 \%, 46$ patients had negative skin prick tests and a further 2 patients' weight was outside the range for dosing. $67 / 510(13.1 \%)$ patients met all the licensing criteria for omalizumab. There has therefore not been any increase in the proportion of eligible patients since the widening of the IgE range. Of these 67, 27 patients had two or more admissions with asthma exacerbations in the previous year. A further 16 patients had one admission but less than $2 \mathrm{~A} \& \mathrm{E}$ attendances. 12 patients required maintenance oral prednisolone but did not have sufficient admissions to meet the NICE prescribing guidelines. In total, 27/510 (5.3\%) of patients with difficult-to-treat asthma were eligible for treatment with omalizumab according to NICE guidance. Although omalizumab is a helpful additional therapy for allergy-mediated asthma, its licensing criteria and current NICE guidelines limits its use in the UK to a small proportion of patients with difficult asthma.

\section{P21 SUCCESS RATE OF SPUTUM INDUCTION IN THE LEICESTER PAEDIATRIC SEVERE ASTHMA CLINIC USING}

doi:10.1136/thx.2010.150961.21

R Herzallah, KS Staley, M Mc Feeters, C Brightling, P Bradding, D Parker, H Pandya, E Humphreys, EA Gaillard. University of Leicester, Leicester, Leicestershire

Background Asthma is a common disease affecting about one in four children in the UK. It is characterised by variable airway obstruction, bronchial hyper responsiveness and influx of inflammatory cells into the bronchial mucosa. However, the mechanism of childhood asthma is still not well understood. Moreover, a subgroup of children with asthma has difficult to treat asthma, defined as persisting symptoms despite maximal conventional therapy. Management decisions in this subgroup are particularly difficult. These patients are often on high doses of steroids, with the attendant risk. A recent study in adults showed that titrating the dose of steroids to sputum eosinophils provided better asthma control without increasing the cumulative doses received by a patient. Neutrophilc airway inflammation was also shown to be a feature of some patients with difficult asthma, which may explain their poor response to corticosteroids. Therefore, knowing the inflammatory cell pattern in our cohort of children with difficult to treat asthma could potentially be a very useful tool in their management. To aid in our understanding and management of our children with difficult asthma we routinely evaluate induced sputum from children over the age of 6 years for the presence of airway inflammatory cells. A dedicated paediatric sputum lab with negative pressure facilities was made available to us by the hospital. Study Aims To review the protocol, success rate and pattern of airway inflammation in our cohort of children with severe asthma. Methods Review of all paediatric sputum inductions performed between March 2008 and February 2010.
Results Between March 2009 and February 2010, 57 sputum inductions were performed on 40 children, $50 \%$ males, between 6 and 18 years of age (median $=12.5$ years). A differential inflammation cell count was obtained from 52 of the 57 samples, giving a success rate of more than $90 \%$. There was no significant difference in the inflammatory cell pattern between those on BTS step 3 and those on BTS steps 4 and $5.42 \%$ of our patients had eosinophila (defined as more than $3 \%$ eosinophils), $31 \%$ had neutrophila (defined as more than $54 \%$ neutrophils), $13 \%$ had both eosinophilia and neutrophilia and only $13 \%$ had a normal inflammatory cell pattern (Abstract P21 Table 1).

\section{Abstract P21 Table 1}

\begin{tabular}{llllll}
\hline & Neutrophils & Monocytes & Eosinophils & Lympocytes & Epithelial \\
\hline Median (\%) & 49.5 & 25.1 & 5.4 & 1.3 & 0.75 \\
Range (\%) & $0-98.5$ & $0-95$ & $0-72.6$ & $0-50.2$ & $0-12.4$ \\
\hline
\end{tabular}

\section{Conclusions}

1. Adequate sputum samples are obtained from most children with severe asthma when performed in a dedicated induced sputum laboratory.

2. A sputum eosinophilia was present in $55 \%$ of children studied with severe asthma.

3. The clinical value of monitoring airway inflammation in children with severe asthma using sputum induction remains to be established.

\section{P22 FACTORS ASSOCIATED WITH REFRACTORINESS TO REPEAT EXERCISE CHALLENGE IN ADULTS WITH ASTHMA}

doi:10.1136/thx.2010.150961.22

N Martin, ID Pavord. Institute for Lung Health, Glenfield Hospital, Leicester, UK

Introduction A proportion of patients with asthma develops a refractory period, during which the bronchoconstrictor response to a second indirect challenge is reduced. A better understanding of factors associated with the development of refractoriness is potentially important as the refractory period may be the vestigial expression of a bronchoprotective pathway that may be pathogenically important and could be manipulated for therapeutic effect. We investigated factors associated with refractoriness in 10 subjects with mild to moderate asthma.

Methods Subjects were recruited from primary care, had mild to moderate (Step 1 or 2 BTS guideline) asthma and were aged 18-50 years. Subjects underwent a standardised laboratory exercise challenge test breathing cold dry air. Those who had a positive test ( $>15 \%$ fall in FEV1) were observed until lung function returned to within $5 \%$ of baseline and then underwent a further standardised exercise challenge test. The refractory index was calculated on the \% reduction in the maximum percent fall in $\mathrm{FEV}_{1}$ between the first and second challenge. On a separate occasion the subjects undertook a sodium metabisulphite challenge test (a known, indirectly acting bronchoconstrictor) and a PD20 (MBS PD20) was calculated for this from interpolation of the log-dose response curve.

Results 22 individuals were screened to find 10 (1F; 9M) with a positive exercise test; these then went on to have a second challenge test. The mean (SEM) fall in FEV1 was 24.9\% (2.4) and 13.7\% (2.5) with first and second challenge respectively. The mean (SEM) refractory index was $50.1 \%$ (6.5). There was no correlation between the refractory index and age, sex, FEV1 \% predicted, FVC, FEV1/ FVC ratio, MBS PD20, or \% fall in FEV1 on first challenge.

Conclusions Our findings suggest that refractoriness is independent of baseline lung function and the magnitude of the response to the initial challenge. Further studies are required to determine whether other demographic factors are associated with this phenomenon. 